\title{
Review of: "Prevalence and antimicrobial susceptibility pattern of urinary tract infection among pregnant women attending Hargeisa Group Hospital, Hargeisa, Somaliland"
}

\section{Taha Gueddi}

Potential competing interests: The author(s) declared that no potential competing interests exist.

The study was done on 422 participants, so in the same time it may give a contractual interpretation but still less than 500, so doesn't give a significant conclusion. In addition to criteria selection. 